\title{
Prognostic Factors in Gastrointestinal Stromal Tumors (GIST): Could Prognostic Nutritional Index (PNI) be a New Prognostic Factor?
}

\author{
Elif Yuce ${ }^{1}$, Celal Alandag ${ }^{2}$, Emel Cakir ${ }^{3}$ and Evren Fidan ${ }^{1}$ \\ ${ }^{1}$ Department of Medical Oncology, Karadeniz Technical University, Trabzon, Turkey \\ ${ }^{2}$ Department of Medical Oncology, Sivas Numune Hospital, Sivas, Turkey \\ ${ }^{3}$ Department of Pathology, Karadeniz Technical University, Trabzon, Turkey
}

\begin{abstract}
Objective: To investigate factors that may affect prognosis in gastrointestinal stromal tumors (GISTs).

Study Design: A descriptive study.

Place and Duration of Study: Karadeniz Technical University Hospital, Trabzon, Turkey from 2000 to 2019.

Methodology: All the patients diagnosed with GIST and followed-up in this centre were included. Those who were not followed-up in this centre were excluded. The Chi-square test for differences between variables in independent groups; and the Kaplan-Meier method for survival rates were used.

Results: Median tumor size was larger in patients with recurrence, compared to those without $(8 \mathrm{~cm}$ vs. $5 \mathrm{~cm}, \mathrm{p}<0.001)$. Recurrence rates were higher with mitosis $\geq 5$ in 50 high-power-fields than with low mitosis $(52.6 \%$ vs. $23.4 \%, p=0.021)$. Median Ki-67 percentages were higher in patients with recurrence than without ( $5 \mathrm{vs} .2, \mathrm{p}=0.031)$. Recurrence rates were higher with necrosis and bleeding than without ( $57.7 \%$ vs. $14.3 \%, p=<0.001 ; 50 \%$ vs. $13.8 \%, p=0.003$ ). Median overall-survival (OS) was shorter in with mitotic counts $\geq 5$ compared to $<5$ (52.0 vs. 110.0 months, $p=0.051)$ and with ulceration than without ( 36.0 vs. 110.0 months, $p=0.017)$. The groups below $(<43.5)$ and above $(>43.5)$ the median prognostic-nutritional-index (PNI) value were similar in terms of OS and disease-free survival (DFS) (52 vs. 70 months, $p=0.174 ; 82$ vs. 100 months, $p=0.411$ ). Median DFS was shorter with ulceration than without (27 vs. 100 months, $p=0.048$ ).

Conclusion: While necrosis, bleeding, ulceration, mitosis, size, and Ki-67 significantly affect the prognosis in GIST, PNI has no significant effect.
\end{abstract}

Key Words: Gastrointestinal stromal tumors (GIST), Survival, Prognosis, Recurrence, Prognostic Nutrition Index (PNI).

How to cite this article: Yuce E, Alandag C, Cakir E, Fidan E. Prognostic Factors in Gastrointestinal Stromal Tumors (GIST): Could Prognostic Nutritional Index (PNI) be a New Prognostic Factor?. J Coll Physicians Surg Pak 2022; 32(01):81-85.

\section{INTRODUCTION}

Gastrointestinal stromal tumors (GISTs) are the most common mesenchymal tumors of the gastrointestinal tract and may be found in all regions of the gastrointestinal tract, but most frequently in the stomach, followed by the small intestine. ${ }^{1} \mathrm{C}$-kit expressionis responsible for the pathogenesis. ${ }^{2}$

Studies have shown that prevalence varies depending on geographical regions..$^{3,4}$ In the most extensive studies related to GISTs, these tumors have more commonly been encountered in the Asian continent, and an Asian-consensus-guideline was published based on the hypothesis that the clinical course may differ from that of GISTs in Europe. ${ }^{5}$

Correspondence to: Dr. Elif Yuce, Department of Medical Oncology, Karadeniz Technical University, Trabzon,

Turkey

E-mail: merevelif@gmail.com

Received: October 16, 2020; Revised: June 05, 2021;

Accepted: September 08, 2021

DOI: https://doi.org/10.29271/jcpsp.2022.01.81
The clinical manifestations are highly variable. They may be benign or malignant GISTs, most commonly exhibit metastasis to the liver. The principal prognostic markers are size $(\mathrm{cm})$, mitotic-count (number of mitoses per 50 high power fields [HPF]), and location. ${ }^{6,7}$

The most extensive study of GISTs was performed by Seker etal. That study investigated 333 patients from nine centres and was intended to identify factors affecting the prognosis, overall-survival (OS) and disease-free-survival (DFS). ${ }^{8} \mathrm{~A}$ similar study was performed in the region, which was not included in Seker's research, and various different results to those of Seker et al. were reported. ${ }^{9}$

Recent studies have shown that nutritional status is closely related to prognosis in many cancers, and malnutrition is very common in cancer patients. ${ }^{10,11}$ Since malnutrition has an important relationship with prognosis, cancer patients should be examined clinically (body mass index and skeletal muscle volume) and in the laboratory in terms of nutritional parameters. The prognostic nutritional index (PNI) is an index used to evaluate the nutritional status of patients in the laboratory. It is 
known that PNI is closely related to prognosis in many cancers. Studies investigating the importance of PNI in GISTs are few. ${ }^{10,12,13}$

Since the clinical and pathological characteristics of GISTs are known to be able to exhibit regional variations, this study was conducted to investigate this variation and improve the approach to GISTs.

The objective of this study was to review the clinicopathological features that affect the prognosis of GISTs; and to investigate whether PNI has an effect on prognosis.

\section{METHODOLOGY}

One-hundred-and-five patients, diagnosed with the pathological diagnosis of GIST at the Karadeniz Technical University Hospital between January 2000 and January 2019, were included in the study. Ethics Committee approval of the study was received from KTU Faculty of Medicine Scientific Research Ethics Committee. Data were obtained from the records. Demographic-data, whether surgical procedures were performed, medical treatment was administered, and if so, the duration thereof, responses to treatment, presence of local-recurrence or distant metastasis, the site of the metastasis, and patients' latest status were recorded. PNI was calculated using albumin-lymphocyte data at time of diagnosis (PNI formula: 0.005 *total-lymphocyte-count $/ \mathrm{mm}^{3}+10 *$ albumin $\mathrm{g} / \mathrm{dl}$ ). Patients were divided into two groups, below and above the median PNI value, after which OS and DFS analysis was performed.

Macroscopic and microscopic characteristics and risk groups were recorded by examining pathology reports. Mitotic-counts were calculated in 50-HPF. The Armed-Forces-Institute of Pathology (AFIP) criteria were used in patient risk classification. The effect on overall and disease-free survival of the data obtained was then examined.

Statistical analysis was performed on statistical package for social sciences (SPSS) version 23.0 software. Descriptive statistics were expressed as numbers and percentages or categorical variables and as mean, median, standard deviation, 25th percentile and 75th percentilefor numerical variables. Compatibility with normal distribution was determined using the OneSample-Kolmogorov-Smirnov test. Since normal distribution conditions were not established, numerical variables were compared between two independent groups using the MannWhitney U- test. The Chi-square test or Fisher's Exact test was used to analyse differences between proportions of categorical variables in independent groups. Survival rates were calculated using the Kaplan-Meier method and log rank test. Alpha significance was setat $p<0.05$.

\section{RESULTS}

The clinical and demographic characteristics of the patients were examined (Table I). The difference in recurrences rates was evaluated in early-stage patients with and without adjuvant-therapy. Fifteen (18.7\%) of the 80 patients whose treatment information was accessible received adjuvant imatinib therapy, while adjuvant therapy was not received by 65 (81.3\%). Recurrence occurred in $18(27.7 \%)$ of the patients not receiving adjuvant therapy, while 35(53.8\%) werefollowed-up without regression, and recurrence data for 12 (18.5\%) could not be evaluated. Recurrence occurred in the form of distant metastasis in $12(66.7 \%)$ of the 18 patients not receiving adjuvant therapy, and locally in 6 (33.3\%). Liver metastasis was detected in 6 $(50 \%)$ of the 12 patients with distant metastasis. Recurrence occurred in $3(20 \%)$ of the patients receiving adjuvant therapy, but not in 9 (60\%), while recurrence data for $3(20 \%)$ couldn't be evaluated. Distant metastasis to the liver occurred in 1 (33.3\%) of the 3 patients with recurrence under adjuvant therapy, and local recurrence in 2 (66.7\%).

Table I: Demographic and clinical characteristics of the patients.

\begin{tabular}{|c|c|}
\hline & n (\%) \\
\hline \multicolumn{2}{|l|}{ Gender: } \\
\hline Male & $44(41.9 \%)$ \\
\hline Female & $61(58.1 \%)$ \\
\hline \multicolumn{2}{|l|}{ Age: } \\
\hline Mean & $62.3 \pm 13.2(\min .23, \max 91)$ \\
\hline Median & 64 \\
\hline \multicolumn{2}{|l|}{ Diagnostic complaint*: } \\
\hline Abdominal pain** & $46(43.8 \%)$ \\
\hline GIS bleeding & $13(12.4 \%)$ \\
\hline Dyspepsia & $6(5.7 \%)$ \\
\hline Change in bowel habits & $5(4.8 \%)$ \\
\hline Abdominal distension & $4(3.8)$ \\
\hline Other & $11(10.5 \%)$ \\
\hline No complaint & $6(5.7)$ \\
\hline Unknown & $14(13.3 \%)$ \\
\hline \multicolumn{2}{|l|}{ Primary localisation: } \\
\hline Stomach & $58(55.2 \%)$ \\
\hline Small Intestine & $29(27.6 \%)$ \\
\hline Colon & $3(2.9 \%)$ \\
\hline Rectum & $4(3.8 \%)$ \\
\hline Retroperitoneal & $2(1.9 \%)$ \\
\hline Intraabdominal & $8(7.6 \%)$ \\
\hline \multicolumn{2}{|l|}{ Surgical status: } \\
\hline Yes & $98(93.3 \%)$ \\
\hline No & $5(4.8 \%)$ \\
\hline Unknown & $2(1.9 \%)$ \\
\hline \multicolumn{2}{|l|}{ Risk Group***: } \\
\hline Low-very low & $43(41.0 \%)$ \\
\hline Medium & $8(7.6 \%)$ \\
\hline High & $29(27.6 \%)$ \\
\hline Insufficient data & $3(2.8 \%)$ \\
\hline Unknown & $22(21.0 \%)$ \\
\hline \multicolumn{2}{|c|}{ Adjuvan treatment status*****: } \\
\hline Yes & $15(16.9 \%)$ \\
\hline No & $65(73.0 \%)$ \\
\hline Unknown: & $9(10.1 \%)$ \\
\hline \multicolumn{2}{|c|}{ Metastasis at diagnosis*****: } \\
\hline Yes & $9(8.6)$ \\
\hline No & $89(84.8 \%)$ \\
\hline Unknown & $7(6.7 \%)$ \\
\hline \multicolumn{2}{|c|}{$\begin{array}{l}\text { *Incidences of diagnostic symptoms reported from } 91 \text { patients with known } \\
\text { symptoms. **Five (10.9\%) of the } 46 \text { patients with abdominal pain } \\
\text { presented with manifestations of acute abdomen. }{ }^{* * *} \text { Risk group } \\
\text { incidences reported from } 83 \text { patients capable of risk group } \\
\text { determination. ****eceipt of adjuvant therapy percentages reported } \\
\text { from } 80 \text { patients whose treatment records were } \\
\text { accessible. }{ }^{* * * * * I n c i d e n c e s ~ o f ~ m e t a s t a s i s ~ a t ~ t i m e ~ o f ~ p r e s e n t a t i o n ~ r e p o r t e d ~} \\
\text { from patients with known metastatic status. }\end{array}$} \\
\hline
\end{tabular}

Patients with and without recurrence were compared in terms of 
size, Ki-67, mitotic-count, macroscopic characteristics, and risk-groups. The findings are summarised in Tablell.

Table II: Comparison of pathologic features and recurrence frequency of GIST.

\begin{tabular}{|c|c|c|c|}
\hline & Recurrence (+) & Recurrence (-) & p-value \\
\hline $\begin{array}{l}\text { Median } \\
\text { tumor size }\end{array}$ & $8(7-18)(n: 21)$ & $5(3-7)(n: 43)$ & $p<0.001$ \\
\hline Median Ki 67 (\%) & $5(3.5-12.5)(n: 18)$ & $2(1-7)(n: 42)$ & p: 0.031 \\
\hline \multicolumn{4}{|c|}{ Mitotic count (in $50 \mathrm{HPF}$ ): } \\
\hline$\geq 5$ & $10(52.6 \%)$ & $9(47.4 \%)$ & \multirow{2}{*}{ p:0.021 } \\
\hline$<5$ & $11(23.4 \%)$ & $36(76.6 \%)$ & \\
\hline \multicolumn{4}{|l|}{ Necrosis: } \\
\hline Yes & $15(57.7 \%)$ & $11(42.3 \%)$ & \multirow{2}{*}{$p<0.001$} \\
\hline No & $5(14.3 \%)$ & $30(85.7 \%)$ & \\
\hline \multicolumn{4}{|l|}{ Ulceration: } \\
\hline Yes & $9(52.9 \%)$ & $8(47.1 \%)$ & \multirow{2}{*}{ p:0.035 } \\
\hline No & $10(24.4 \%)$ & $31(75.6 \%)$ & \\
\hline \multicolumn{4}{|l|}{ Bleeding: } \\
\hline Yes & $15(50.0 \%)$ & $15(50.0 \%)$ & \multirow{2}{*}{ p:0.003 } \\
\hline No & $4(13.8 \%)$ & $25(86.2 \%)$ & \\
\hline \multicolumn{4}{|l|}{ Risk Group: } \\
\hline Low & $4(12.1 \%)$ & $29(87.9 \%)$ & \multirow{2}{*}{ p:0.001 } \\
\hline High & $10(58.8 \%)$ & $7(41.2 \%)$ & \\
\hline
\end{tabular}

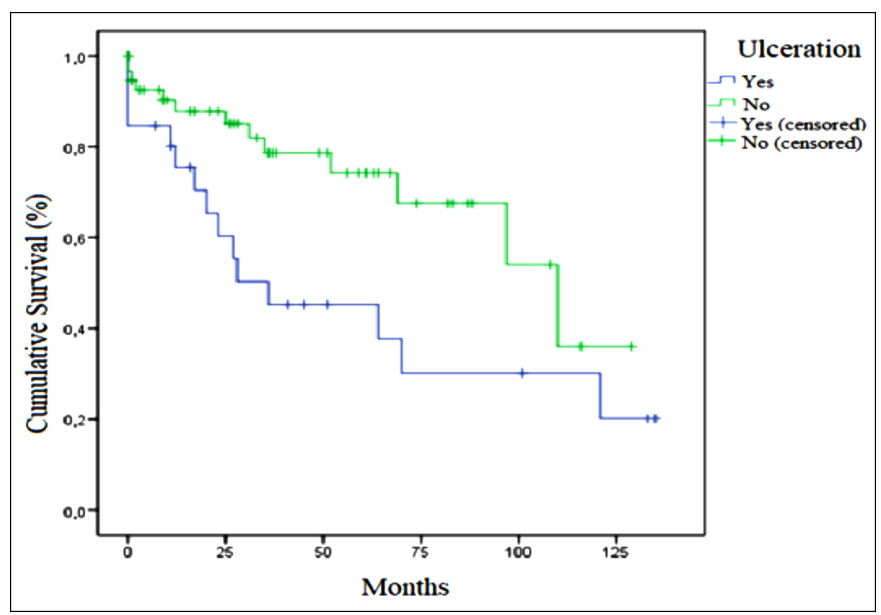

Figure 1: Relation of ulceration and overall survival in GIST.

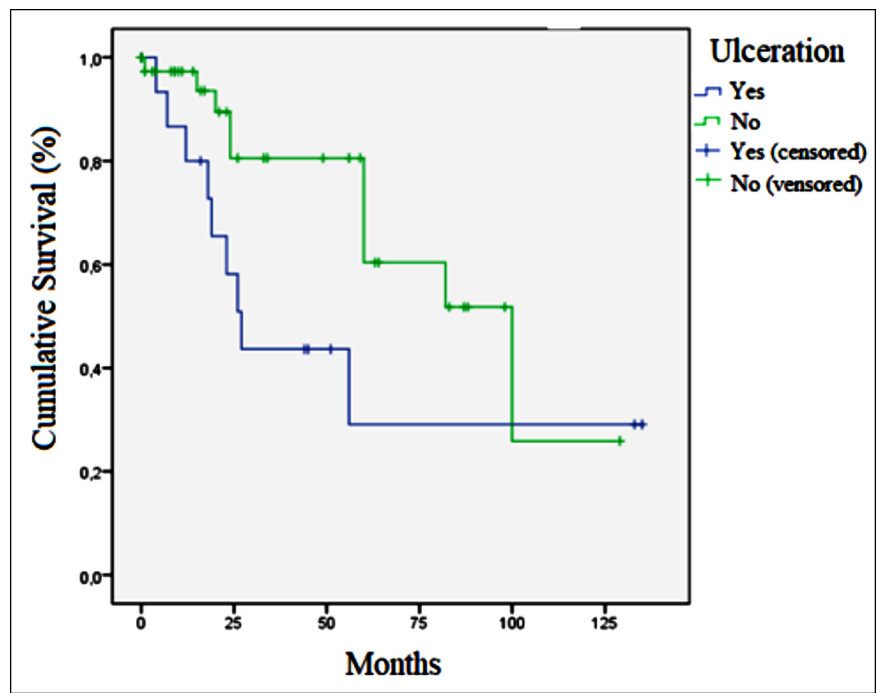

Figure 2: Relation of ulceration and disease free survival in GIST.

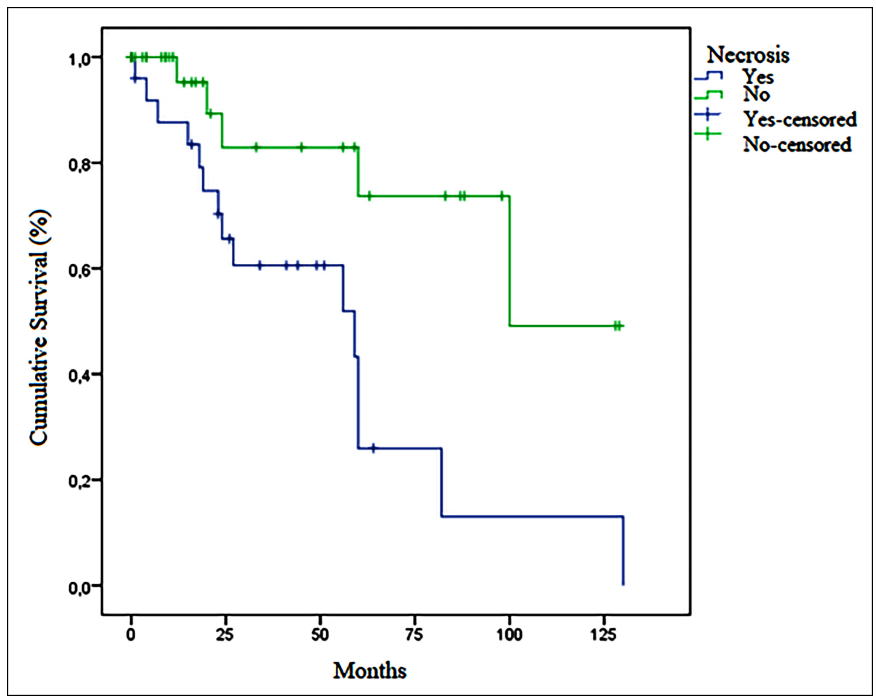

Figure 3: Relation of necrosis and disease free survival in GIST.

Mitotic-count was $\geq 5$ (in $50-\mathrm{HPF}$ ) in $5(71.4 \%$ ) patients of metastatic patients at the time of diagnosis, and in 26 (30.2\%) patients of non-metastatic patients $(p=0.039)$. No significant difference was determined between metastatic and non-metastatic patients at time of diagnosis in terms of size or Ki-67 ( $p=$ 0.057 and $p=0.064$, respectively).

Factors affecting survival in GISTs were also examined. Median OS was 52.0 months in patients with mitotic-counts $\geq 5$ in 50HPF and110.0 months in those with counts $<5$ ( $p=0.051)$. No difference in OS was determined between patients with tumor sizes $\leq 5 \mathrm{~cm}$ and $>5 \mathrm{~cm}$ ( $93 \mathrm{vs}$. 67 months, $p=0.957$ ). Median OS in individuals with ulceration was 36.0 months, compared to 110.0 months in patients without ulcerations $(p=0.017$, Figure 1). Median DFS was 27.0 months $(95 \% \mathrm{Cl}, 19.8-34.1)$ in patients with ulceration and 100 months $(95 \% \mathrm{Cl}, 55.9-144.0)$ in those without ulceration ( $p=0.048$, Figure 2 ). No difference in survival was observed between patients with necrosis or bleeding and those without ( $p=0.293$ and $p=0.544$, respectively). Median OS was 93.0 months in the low-risk group, and 36.0 months in the moderate-high risk group $(p=0.040)$. Median OS in metastatic patients at time of diagnosis was 17 months $(95 \% \mathrm{Cl}, 0-43.3)$, compared to 93.0 months $(95 \% \mathrm{Cl}$, $57.9-128.1)$ in non-metastatic patients $(p=0.019)$. Median DFS was statistically significantly shorter in patients with necrosis than in those without ( $59.0 \mathrm{vs} .100 .0$ months, $p=0.007$ ), Figure 3). No significant difference in OS was observed in terms of gender, primary localisation, receipt of adjuvant therapy, or Ki-67 ( $p=0.250, p=0.945, p=0.718$, and $p=0.411$, respectively). No significant difference was also observed in groups with below-median PNI values $(<43.5)$ and above-median values (>43.5) in terms of OS (52 vs. 70 months, $p=0.174$ ) or DFS (82 vs. 100 months, $p=0.411$ ).

\section{DISCUSSION}

The median tumor size in this study was higher in patients with recurrence $(8 \mathrm{~cm}$ vs. $5 \mathrm{~cm}, \mathrm{p}<0.001)$. Recurrence rates were also higher in patients with mitotic-counts $\geq 5$ in $50-\mathrm{HPF}$ 
compared to those with values $<5$ ( $52.6 \%$ vs. $23.4 \%, p=0.021)$. These findings suggest that tumor size and mitotic-count are powerfully associated with tumor proliferative activity and the risk of recurrence. Although we observed no difference in OS between patients with tumor sizes $\leq 5 \mathrm{~cm}$ and $>5 \mathrm{~cm}$, median OS was shorter in patients with mitotic counts $\geq 5$ in 50 -HPF than in those with counts $<5$. The significantly shorter OS times in patients with high mitotic-counts confirm the importance of these two parameters in prognostic screening.

Some studies have proposed the prognostic significance of Ki-67 in GIST. 9,14,15 The Ki-67 percentage in the present study was higher in patients with recurrence than in those without (5 vs. 2, $p=0.031$ ). Although the mean Ki-67 percentage in patients with recurrence was higher than in those without recurrence, there was no difference in terms of OS between patients with $\mathrm{Ki}-67$ percentages $<5$ and $>5$. Although the findings suggest that the Ki-67 percentage may be important in prognosis, more extensive studies are needed.

There are thought to be other pathological prognostic indicators in GISTs in addition to size and mitotic count. Based on the thesis that necrosis is associated with the general proliferative activity of the tumor, studies have suggested that prognosis may be poorer in tumors with necrosis with a macroscopic

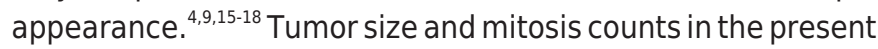
study were also higher in necrotic tumors $(p<0.001$ and $p=$ 0.003 , respectively). Recurrence occurred in $57.7 \%$ of necrotic tumors and in $14.3 \%$ of non-necrotic tumors ( $p<0.001)$. Median DFS was statistically significantly shorter in patients with necrosis than in those without (59.0 vs. 100.0 months, $p=$ 0.007 , Figure 3 ). This study therefore supports the idea that necrosis may be prognostic in GISTs. Tumor size, mitosis counts, and Ki-67 levels were also statistically significantly higher in tumors with a macroscopic bleeding appearance in this study ( $p<0.001, p=0.007$ and $p=0.022$, respectively). Recurrence occurred in $50 \%$ of tumors with bleeding and in $13.8 \%$ of those without ( $p=0.003$ ). Necrosis and bleeding in a tumor may be associated with a large tumor size and high mitotic activity, and that recurrence may therefore be more frequent in such tumors, may improve the management of these patients. In addition, the mitotic count was significantly higher in tumors with ulceration in this study $(p=0.001)$. Although no statistically significant difference was observed in terms of prevalence of recurrence, OS and DFS were significantly shorter in tumors with ulceration (median OS 36 vs. 110 months, $p=0.017$ and median DFS 27 vs. 100 months, $p=$ 0.048 , respectively, Figures 1 and 2 ). In the light of these findings it should be remembered that macroscopic pathological features in GISTs may be closely associated with prognosis, and surgeons and pathologists should pay attention to the macroscopic characteristics of the tumor, and oncologists should bear these in mind when planning treatment.

Studies have reported that the primary localisation of GISTs can affect survival. Although survival is better in gastric tumors, one study from the Thrace region in Turkey reported better prog- nosis in small intestinal tumors. ${ }^{7,9}$ In addition, in a study published in 2005, Nakamura et al. reported no difference in survival between gastric and non-gastric GISTs. ${ }^{19}$ No significant effect of primary localisation on survival was also detected in this study. These findings may derive from the low patient number as well as from the clinical features of GISTs exhibiting regional variation.

PNI is an easy yet efficient index using serum albumin level and lymphocyte counts in peripheral blood. Although its relationship with prognosis has been demonstrated in various cancer types, it is particularly important in gastrointestinal tract tumors. ${ }^{20,21}$ Few studies are investigating the effect of PNI on GISTs. In a study published in 2019 on 431 patients in China, it was shown that patients with a high PNI group had higher recurrence-free survival (RFS) rates than those in the low group (5-year RFS rate $89.9 \%$ versus $70.8 \%, p<0.001$ ) and PNI was found to be an independent prognostic factor of RFS $(p=0.004) .{ }^{22}$ Again, in a study of 206 patients conducted in China, it was shown that RFS wasstatistically significantly better in patients in the high PNI group. Also the effects of PNI, platelet-to-lymphocyte ratio (PLR), and neutrophyl-to-lymphocyte ratio (NLR) on prognosis were investigated and they showed that only preoperative high PNI is an independent prognostic factor for survival $(p=0.031) .{ }^{13}$

Since previous studies have shown that the PNI affects prognosis in various cancer patients, the authors also investigated the effect of this index on OS and DFS $(10,11,13)$. No difference in OS or DFS was detected between the groups below and above the median PNI value. These results may be due to GISTs being relatively less common than more frequently seen cancers such as lung, breast and kidney cancer, and thus to our low patient number.

To the best of authors' knowledge, this study is one of the first studies investigating the relationship between prognostic nutritional index (PNI) and GIST. Since the clinical and pathological characteristics of GISTs are known to exhibit regional variation, the authors think that those of the findings that differ from the previous literature should be borne in mind in addition to those in general agreement with previous studies.

\section{CONCLUSION}

The clinical characteristics of GISTs may vary depending on geographical regions. Findings affecting prognosis in GISTs include necrosis, bleeding and ulceration, in addition to mitosis counts, tumor size and Ki-67 percentage. PNI may not affect prognosisin GIST.

\section{ETHICALAPPROVAL:}

Ethics Committee approval of the study was received from KTU Faculty of Medicine Scientific Research Ethics Committee on 4.3.2019. (Document No. 24237859-214, approval No. 2019/37).

\section{PATIENTS' CONSENT:}

Patients' consent is not required because this is a retrospective study. 


\section{CONFLICT OF INTEREST:}

The authors declared no conflict of interest.

\section{AUTHORS' CONTRIBUTION:}

EY: Conceptualisation, methodology, validation, writing originaldraft, visiusalition.

CA: Statistical analysis, investigation.

EC: Pathological examination, data curation.

EF: Writing review-editing, supervision.

\section{REFERENCES}

1. Miettinen M, Lasota J. Gastrointestinal stromal tumors: Review on morphology, molecular pathology, prognosis, and differential diagnosis. Arc Pathol LabMed 2006; 130 (10):1466-78. doi: 10.5858/2006-130-1466-GSTROM.

2. Hirota S, Isozaki K, Moriyama Y, Hashimoto K, Nishida T, Ishiguro S, et al. Gain-of-function mutations of c-kit in human gastrointestinal stromal tumors. Sci 1998; 279 (5350):577-80. doi: 10.1126/science.279.5350.577.

3. Søreide K, Sandvik OM, Søreide JA, Giljaca V, Jureckova A, Bulusu VR. Global epidemiology of gastrointestinal stromal tumours (GIST): A systematic review of population-based cohort studies. Cancer Epidemiol 2016; 40: 39-46. doi: 10.1016/j.canep.2015.10.031.

4. Yi M, Xia L, Zhou Y, Wu X, Zhuang W, Chen Y, et al. Prognostic value of tumor necrosis in gastrointestinal stromal tumor: A meta-analysis. Medicine (Baltimore) 2019; 98(17):e15338. doi: 10.1097/MD.0000000000015338.

5. Koo DH, Ryu MH, Kim KM, Yang HK, Sawaki A, Hirota S, et al. Asian consensus guidelines for the diagnosis and management of gastrointestinal stromal tumor. Cancer Res Treat 2016; 48(4):1155-66. doi=10.4143/crt.2016.187

6. Joensuu $H$, Vehtari A, Riihimäki J, Nishida T, Steigen SE, Brabec $\mathrm{P}$, et al. Risk of recurrence of gastrointestinal stromal tumour after surgery: An analysis of pooled population-based cohorts. Lancet Oncol 2012; 13(3): 265-74. doi: 10.1016/S1470-2045(11)70299-6.

7. Miettinen M, El-Rifai W, Sobin L, Lasota J. Evaluation of malignancy and prognosis of gastrointestinal stromal tumors: A review. Hum Pathol 2002; 33(5):478-83. doi: 10.1053/hupa.2002.124123.

8. Seker M, Sevinc A, Yildiz R, Cihan S, Kaplan MA, Gokdurnali $A$, et al. Prognostic factors in gastrointestinal stromal tumors: Multicenter experience of 333 cases from Turkey. Hepatogastroenterology 2013; 60(124):768-75. doi: 10.5754/hge11666.

9. Merev E, Cicin I. Effect of clinical and pathological features of gastrointestinal stromal tumors on overall survival and prognosis: Single centre experience. J Oncol Sci 2019; http://linkinghub.elsevier.com/retrieve/pii/S2452336419300 536.

10. Yasar HA, Bir Yucel K, Arslan C, Ucar G, Karakaya S, Bilgin $B$, et al. The relationship between prognostic nutritional index and treatment response in patients with metastatic renal cell cancer. J Oncol Pharm Pract 2019; 26(5):
1110-16. doi/10.1177/1078155219883004.

11. Hu Y, Shen J, Liu RK, Feng ZM, Zhang CN, Ling L, et al. Prognostic value of pretreatment prognostic nutritional index in non-small cell lung cancer: A systematic review and meta-analysis. Int J Biol Markers 2018; 33(4):372-8. doi: 10.1177/1724600818799876.

12. Lin $Y$, Wang $M$, Jia J, Wan W, Wang T, Yang W, et al. Development and validation of a prognostic nomogram to predict recurrence in high-risk gastrointestinal stromal tumour: A retrospective analysis of two independent cohorts. EBioMedicine 2020; 60:103016. doi: 10.1016/j. ebiom.2020.103016.

13. Shi WK, Zhang XH, Zhang J, Yu M, Yuan YJ, Xiong W. Predictive ability of prognostic nutritional index in surgically resected gastrointestinal stromal tumors: A propensity score matching analysis. Jpn J Clin Oncol 2019; 49(9): 823-31. doi: 10.1093/jjco/hyz078.

14. Liu LC, Xu WT, Wu X, Zhao P, Lv YL, Chen L. Overexpression of carbonic anhydrase II and Ki-67 proteins in prognosis of gastrointestinal stromal tumors. World J Gastroenterol 2013; 19(16):2473-80. doi: 10.3748/wjg.v19.i16.2473.

15. Basilio-De-Oliveira RP, Pannain VLN. Prognostic angiogenic markers (endoglin, VEGF, CD31) and tumor cell proliferation (Ki67) for gastrointestinal stromal tumors. World J Gastroenterol 2015; 21(22):6924-30. doi: 10. 3748/wjg.v21.i22.6924.

16. Hou YY, Lu SH, Zhou Y, Qi WD, Shi Y, Tan YS, et al. Stage and histological grade of gastrointestinal stromal tumors based on a new approach are strongly associated with clinical behaviors. Mod Pathol 2009; 22(4):556-69. doi: 10.1038/modpathol.2009.11.

17. Kukar M, Kapil A, Papenfuss W, Groman A, Grobmyer SR, Hochwald SN. Gastrointestinal stromal tumors (GISTs) at uncommon locations: A large population based analysis. J Surg Oncol 2015; 111(6):696-701. doi: 10.1002/jso.23873.

18. Oliveira RP, Portari Filho PE, Iglesias AC. Comparative study of the different degrees of risk of gastrointestinal stromal tumor. Rev Col Bras Cir 2015; 42(1):32-6. doi: 10.1590/ 0100-69912015001007.

19. Nakamura N, Yamamoto $\mathrm{H}$, Yao T, Oda $\mathrm{Y}$, Nishiyama KI, Imamura $\mathrm{M}$, et al. Prognostic significance of expressions of cell-cycle regulatory proteins in gastrointestinal stromal tumor and the relevance of the risk grade. Hum Pathol 2005; 36(7):828-37. doi: 10.1016/j.humpath.2005.03.012.

20. Kanda M, Fujii T, Kodera Y, Nagai S, Takeda S, Nakao A. Nutritional predictors of postoperative outcome in pancreatic cancer. BrJ Surg 2011; 98(2):268-74. doi: 10.1002/bjs.7305.

21. Migita K, Takayama T, Saeki K, Matsumoto S, Wakatsuki K, Enomoto $\mathrm{K}$, et al. The prognostic nutritional index predicts long-term outcomes of gastric cancer patients independent of tumor stage. Ann Surg Oncol 2013; 20(8):2647-54. doi: 10.1245/s10434-013-2926-5

22. Sun J, Mei Y, Zhu Q, Shou C, Tjhoi WEH, Yang W, et al. Relationship of prognostic nutritional index with prognosis of gastrointestinal stromal tumors. J Cancer 2019; 10(12): 2679-86. doi: 10.7150/jca.32299. 